\title{
Caracterização físico-química de polpa e casca de pitaya 'Golden'
}

\author{
Physicochemical characterization of 'Golden' pitaya pulp and bark \\ Caracterización fisicoquímica de la pulpa y corteza de pitaya 'Golden'
}

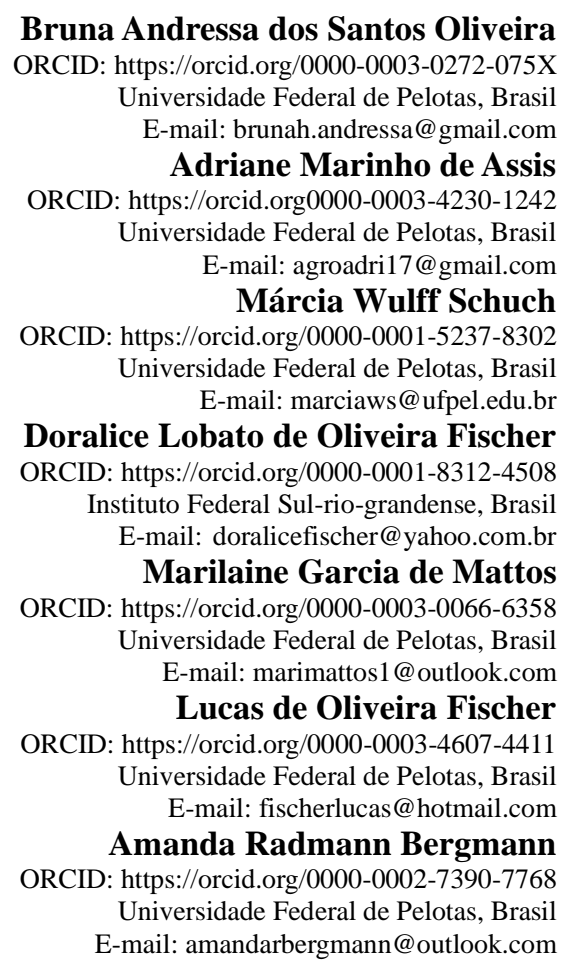

\begin{abstract}
Resumo
Com este trabalho, teve-se por objetivo avaliar as características físico-químicas da polpa e da casca de pitaya 'Golden'. O delineamento experimental foi constituído em esquema unifatorial, com dois níveis (polpa e casca), utilizando dez repetições de um fruto cada. Foram avaliadas as características físico-químicas mencionadas a seguir. Para sólidos solúveis e pH, a polpa e a casca apresentaram valores médios de 8,31 e 4,50 ${ }^{\circ}$ brix e de 4,79 e 5,02 respectivamente. Em relação à acidez titulável, a casca e a polpa apresentaram médias de 0,34 \% e 0,18\% de ácido málico. Para a massa média dos frutos, massa da polpa e da casca obteve-se valores médios de 360,45g, 264,04g e 95,63 g, respectivamente. Em relação as variáveis comprimento dos frutos, comprimento da polpa, diâmetro do fruto e diâmetro da polpa, observou-se $138,65 \mathrm{~mm}, 98,67 \mathrm{~mm}, 73,58 \mathrm{~mm}$ e $63,78 \mathrm{~mm}$, respectivamente. Quanto a firmeza da casca e da polpa, o valor médio foi de 9,38 N e 5,89 N, respectivamente. Na espessura da casca, verificou-se média de 2,98 mm. Quanto luminosidade e ângulo hue, observou-se valores médios de 61,68 e 81,79 para casca e polpa, respectivamente. Já para a polpa, os valores médios foram de 35,04 para variável luminosidade e 83,36 para o ângulo hue. Concluiu-se que a polpa e a casca da pitaya 'Golden' apresentam características físico-químicas de acordo com a literatura, possuindo potencial tanto para o consumo in natura quanto para a utilização na indústria, bem como proporções de tamanho adequadas para o mercado externo.
\end{abstract}

Palavras-chave: Hylocereus undatus x Hylocereus undatus; Pitaya amarela; Cactaceae.

\begin{abstract}
The objective of this work was to evaluate the physicochemical characteristics of the pulp and bark of pitaya 'Golden'. The experimental design consisted of a unifactorial scheme, with two levels (pulp and peel), using ten replications of one fruit each. The physicochemical characteristics mentioned below were evaluated. For soluble solids and $\mathrm{pH}$, pulp and skin had mean values of 8,31 and 4,50 ${ }^{\circ}$ brix and 4,79 and 5,02 respectively. Regarding the titratable acidity, the peel and pulp had averages of $0,34 \%$ and $0,18 \%$ of malic acid. For the average fruit mass, pulp and skin mass, average values of 360,45g, 264,04g and 95,63g were obtained, respectively. Regarding the variables fruit length, pulp length, fruit diameter and pulp diameter, it was observed $138,65 \mathrm{~mm}, 98,67 \mathrm{~mm}, 73,58 \mathrm{~mm}$ and $63,78 \mathrm{~mm}$, respectively. As for peel and pulp firmness, the average value was $9,38 \mathrm{~N}$ and 5,89 N, respectively. In the thickness of the shell, there was
\end{abstract}


an average of 2,98 mm. As for luminosity and hue angle, mean values of 61,68 and 81,79 were observed for peel and pulp, respectively. As for the pulp, the average values were 35,04 for variable brightness and 83,36 for the hue angle. It was concluded that the pulp and bark of pitaya 'Golden' have physicochemical characteristics according to the literature, having potential both for fresh consumption and for industrial use, as well as adequate size proportions for the foreign market.

Keywords: Hylocereus undatus $x$ Hylocereus undatus; Yellow pitaya; Cactaceae.

\section{Resumen}

El objetivo de este trabajo fue evaluar las características fisicoquímicas de la pulpa y corteza de pitaya 'Golden'. El diseño experimental consistió en un esquema unifactorial, con dos niveles (pulpa y piel), utilizando diez réplicas de un fruto cada una. Se evaluaron las características fisicoquímicas que se mencionan a continuación. Para los sólidos solubles y el $\mathrm{pH}$, la pulpa y la piel tuvieron valores medios de 8,31 y 4,50 ${ }^{\circ}$ brix y 4,79 y 5,02 respectivamente. En cuanto a la acidez titulable, la cáscara y pulpa tuvieron promedios de $0.34 \%$ y $0.18 \%$ de ácido málico. Para la masa promedio de fruto, pulpa y masa de piel se obtuvieron valores promedio de $360.45 \mathrm{~g}, 264.04 \mathrm{~g}$ y $95.63 \mathrm{~g}$, respectivamente. En cuanto a las variables longitud del fruto, longitud de la pulpa, diámetro del fruto y diámetro de la pulpa, se observó 138,65 mm, 98,67 mm, 73,58 $\mathrm{mm}$ y $63,78 \mathrm{~mm}$, respectivamente. En cuanto a la firmeza de la cáscara y la pulpa, el valor promedio fue de 9,38 N y 5,89 N, respectivamente. En el grosor del caparazón, hubo un promedio de 2,98 mm. En cuanto a luminosidad y ángulo de tono, se observaron valores medios de 61,68 y 81,79 para piel y pulpa, respectivamente. En cuanto a la pulpa, los valores medios fueron 35,04 para el brillo variable y 83,36 para el ángulo de tono. Se concluyó que la pulpa y corteza de pitaya 'Golden' tienen características fisicoquímicas según la literatura, teniendo potencial tanto para consumo fresco como para uso industrial, así como proporciones de tamaño adecuadas para el mercado externo.

Palabras clave: Hylocereus undatus $x$ Hylocereus undatus; Pitaya amarilla; Cactaceae.

\section{Introdução}

A pitaya é uma planta rústica da família Cactaceae, conhecida mundialmente como "Dragon Fruit" (Fruta-do-Dragão), sendo nativa do sul do México e América Central (Lima, 2013; Mercado-Silva, 2018). No Brasil, espécies de Hylocereus e Selenicereus, passaram a ser cultivadas em escala comercial a partir de 1990, devido à crescente demanda e vem ganhando mais espaço em todo o território nacional (Mello, 2014).

Visando atender a novas demandas e ofertar novos sabores, cores e texturas ao mercado interno e externo (Sarmento, 2017), novas cultivares estão sendo introduzidas no mercado, dentre os quais está a pitaya 'Golden', híbrido interclonal (Hylocereus undatus $x$ Hylocereus undatus) de polpa branca com casca amarela (Abdi; Mizrahi, 2012) e sem a presença de espinhos (Mizrahi, 2015).

Devido ao seu sabor doce e suave, sua polpa firme e suas propriedades funcionais, a pitaya é um fruto aceito e valorizado nos mercados consumidores, o que têm despertado o interesse dos muitos produtores (Junqueira et al., 2002), sendo possível realizar o aproveitamento de todas as partes da planta para consumo, como os cladódios, as flores e os frutos (Silva, 2014).

A polpa da pitaya é a parte comercialmente mais nobre (Santos et al., 2015), constituindo 70-80\% da fruta. Pode ser consumida tanto ao natural como transformada numa gama de produtos industrializados, como sorvetes, geleias, sucos, caldas e doces (Gunasena et al., 2007). Inclusive, as sementes também podem ser aproveitadas para extração de óleo (Santos et al., 2015).

Em relação à casca, que corresponde a aproximadamente $33 \%$ do peso total do fruto da pitaya, esta muitas vezes é descartada durante o processamento (Amid; Manap, 2014), embora haja estudos direcionados para investigação de suas possíveis aplicações e propriedades bioativas (Tenore; Novellino; Basile, 2012; Abreu, 2012). Dessa forma, a casca pode ser reaproveitada para a elaboração de corantes de produtos alimentícios (Mello et al., 2015), na produção de farinha (Surdo, 2019; Utpott, 2018), bebidas fermentadas (Sá et al., 2015), geleia (Oliveira et al., 2017), entre outros.

Com base nesses aspectos, a determinação das características físico-químicas dos frutos é de grande importância para o correto direcionamento no mercado, bem como para estabelecer seu valor comercial. Além disso, conforme a espécie, pode 
haver diversificação quanto ao formato, presença de espinhos, cor da casca e da polpa, teores de sólidos solúveis e pH na polpa, reflexo da alta diversidade genética desta frutífera (Lima, 2013), o que poderá interferir nas preferências do consumidor.

Apesar do seu grande potencial comercial, ainda são escassas as informações referentes a caracterização da polpa e da casca, bem como seu potencial para a indústria alimentícia. Em função disso, com este trabalho, objetivou-se avaliar as características físico-químicas da polpa e da casca de uma cultivar de pitaya.

\section{Metodologia}

O experimento foi realizado em maio de 2019, no LabAgro/Fruticultura, no Departamento de Fitotecnia, da Faculdade de Agronomia Eliseu Maciel, na Universidade Federal de Pelotas, localizado no município de Capão do Leão-RS.

Foram utilizados frutos de pitaya 'Golden', provenientes de pomar comercial, com plantas de três anos de idade, localizado em Turvo-SC. Após a colheita manual, os frutos foram acondicionados em caixas térmicas e encaminhados para o laboratório, onde foram analisadas um dia após a colheita.

O delineamento experimental foi constituído em esquema unifatorial (Pitaya 'Golden'), com dois níveis (polpa e casca), com dez repetições de um fruto cada. A separação da polpa e da casca foi efetuada manualmente e, posteriormente foram distribuídas em bandejas plásticas identificadas para a realização das análises.

Quanto as características físicas, foram analisadas: massa (g) total do fruto; massa (g) da polpa e massa (g) da casca; comprimento e diâmetro $(\mathrm{mm})$ do fruto e da polpa; espessura $(\mathrm{mm})$ e firmeza da casca e da polpa; e cor da casca e da polpa. Para a massa total do fruto, da polpa e da casca utilizou-se balança de precisão URANO ${ }^{\circledR}$; comprimento e diâmetro do fruto e da polpa e espessura da casca foram realizados com o auxílio de paquímetro digital Mitutoyo ${ }^{\circledR}$. Com relação à firmeza, utilizou-se penetrômetro tr$^{-I t a l y}{ }^{\circledR}$ com os resultados expressos em Newton (N).

A análise colorimétrica, foi realizada na parte externa da casca e da polpa e determinada com auxílio de um colorímetro Konica Minolta CR 400, utilizando o espaço de cor $\mathrm{L}^{*} \mathrm{a}^{*} \mathrm{~b}^{*}$, também conhecido como espaço de cor CIELAB, onde a coordenada $L^{*}$ indica a luminosidade, a coordenada a* indica vermelho/verde (-a é verde/ + a é vermelho) e a b* é a coordenada amarelo/azul (-b é azul/ +b é amarelo) (Minolta, 2007).

As análises químicas realizadas em triplicata foram: sólidos solúveis (SS); pH e acidez titulável (AT). Os SS foram determinados utilizando-se refratômetro digital $\mathrm{ATAGO}^{\circledR}$, com o resultado expresso em ${ }^{\circ} \mathrm{Brix}$; o pH determinado com peagâmetro $\mathrm{AZ}^{\circledR}$ (Modelo 86505); e a acidez titulável (AT), determinada pelo método de titulometria, utilizando $10 \mathrm{~mL}$ da amostra diluída em $90 \mathrm{~mL}$ de água destilada e a titulação feita com solução de $\mathrm{NaOH} 0,1 \mathrm{~N}$, com auxílio de pHmetro até se atingir pH 8,2, e os resultados expressos em porcentagem de ácido málico (Instituto Adolfo Lutz, 1985).

Para os dados obtidos, foram determinadas as médias, o valor mínimo e máximo, e o coeficiente de variação, por meio do programa estatístico Sisvar ${ }^{\circledR}$.

\section{Resultados e Discussão}

As características físicas da polpa e casca estão descritas na Tabela 1. Para a variável massa média dos frutos, obtevese $360,45 \mathrm{~g}$, enquanto a massa da polpa foi de $264,04 \mathrm{~g}$ e a da casca, de $95,63 \mathrm{~g}$ (Tabela 1).

De acordo com Cordeiro et al. (2015), a pitaya tem muita polpa, quando comparada a outros cactos, sendo um índice qualitativo interessante para a indústria de processamento. Porém, analisando os resultados do presente trabalho observa-se que mesmo apresentando mais que o dobro de polpa com relação à casca, ainda assim 26,54\% da massa do fruto corresponde a massa da casca; logo, esta é a porcentagem descartada quando os frutos de pitaya são destinados ao processamento ou quando são consumidos in natura, ou seja, quando a casca não é aproveitada. 
Tabela 1. Características físicas de pitaya 'Golden’. Pelotas-RS, 2017.

\begin{tabular}{lcccc}
\hline \multicolumn{1}{c}{ Variáveis analisadas } & Média & Mínimo & Máximo & CV (\%) \\
\hline Massa total do fruto $(\mathrm{g})$ & 360,45 & 230,37 & 487,86 & 26,08 \\
Massa da polpa $(\mathrm{g})$ & 264,04 & 158,49 & 370,01 & 29,79 \\
Massa da casca $(\mathrm{g})$ & 95,63 & 71,19 & 117,84 & 18,39 \\
Comprimento do fruto (mm) & 138,65 & 118,02 & 154,09 & 9,31 \\
Diâmetro do fruto (mm) & 73,58 & 60,96 & 82,86 & 10,90 \\
Comprimento da polpa (mm) & 98,67 & 80,92 & 114,52 & 11,02 \\
Diâmetro da polpa (mm) & 63,78 & 52,62 & 74,93 & 11,26 \\
Firmeza da casca (N) & 9,38 & 5,22 & 13,85 & 26,86 \\
Firmeza da polpa (N) & 5,89 & 4,98 & 6,84 & 0,68 \\
Espessura da casca (mm) & 2,98 & 2,40 & 3,88 & 14,79 \\
\hline
\end{tabular}

CV (\%): coeficiente de variação.

Fonte: Bruna Andressa dos Santos Oliveira.

A média registrada para o comprimento do fruto foi de $138,65 \mathrm{~mm}$ e diâmetro de $73,58 \mathrm{~mm}$; enquanto na análise da polpa foram verificados valores de comprimento e diâmetro de 98,67 e 63,78 mm, respectivamente (Tabela 1). Consta na literatura que os frutos da pitaya para exportação devem ter comprimento mínimo de 80 mm e diâmetro de 50 mm (Schwentesius et al., 1999). Além disso, os frutos que apresentam boas características com potencial para o mercado podem ser úteis para programas de seleção, comercialização e melhoramento genético de novas cultivares (Campos - Rojas et al., 2011).

Para a firmeza da casca e da polpa, o valor médio foi de 9,38 N e 5,89 N, respectivamente (Tabela 1). Os resultados observados por Yah et al. (2008) corroboram com este trabalho, em que estudando as características de frutos de pitaya vermelha de polpa branca, verificaram firmeza da polpa de $6,3 \mathrm{~N}$.

Em trabalho realizado por Abdi e Mizrahi (2013) com a pitaya 'Golden', os autores verificaram que os frutos são firmes e que a fruta pode permanecer comerciável após até sete dias de armazenamento em temperatura ambiente, apesar de alguns defeitos visuais. Rosales-Bustamante et al. (2009) relatam que a firmeza do fruto da pitaya é um critério de seleção para o manejo pós-colheita, visto que o tamanho dos frutos e a durabilidade pós-colheita são características que favorecem a venda e a comercialização.

Em relação à espessura da casca, verificou-se média de 2,98 mm (Tabela 1). Em trabalho realizado por Souza et al. (2006), foi observado o valor médio de 3,88 mm de espessura da casca para pitaya (Hylocereus undatus). Conforme Magalhães (2017), a casca da fruta tem um importante fator do ponto de vista pós-colheita, pois protege contra perdas de água e danos mecânicos, químicos e biológicos. Assim, epidermes mais finas podem resultar em frutas mais suscetíveis a danos. Por outro lado, quando mais espessas tendem a reduzir o rendimento da polpa, por ser uma variável de relação.

Quanto à coloração dos frutos, foram observados valores médios de luminosidade e ângulo hue para a casca do fruto de 61,68 e 81,79, respectivamente, indicando a coloração amarela típica da pitaya 'Golden' (Tabela 2). 
Tabela 2. Características colorimétricas de pitaya 'Golden'. Pelotas-RS, 2017.

\begin{tabular}{lcccc}
\hline \multicolumn{1}{c}{ Variáveis analisadas } & Média & Mínimo & Máximo & CV $(\%)$ \\
\hline Luminosidade da casca & 61,68 & 56,02 & 68,51 & 6,07 \\
Hue casca $\left({ }^{\circ}\right)$ & 81,76 & 80,13 & 85,37 & 1,87 \\
Luminosidade da polpa & 35,04 & 21,10 & 47,97 & 21,44 \\
Hue polpa $\left({ }^{\circ}\right)$ & 83,36 & 73,96 & 88,95 & 4,98 \\
\hline
\end{tabular}

CV (\%): coeficiente de variação.

Fonte: Bruna Andressa dos Santos Oliveira.

Para Queiroz e Treptow (2006), a luminosidade é definida como atributo de qualidade das superfícies dos materiais responsável pelas aparências brilhantes ou lustrosas, estando associado à reflexão espectral. É, portanto, uma propriedade relativa, indicando a maneira pela qual a luz é refletida e transmitida, sendo um atributo de aparência e importante para aceitação do produto.

A coloração dos frutos também pode ser interpretada a partir dos dados do ${ }^{\circ}$ hue, o qual expressa a cor propriamente dita do fruto (Moreno et al., 2016). Este é um importante requisito de qualidade na aceitação do produto pelo consumidor e na determinação dos índices de maturação para indicar o ponto ideal de colheita dos frutos (Rangel Junior et al., 2021).

Para a polpa, os valores médios foram de 35,04 para variável luminosidade e 83,36 para o ângulo hue (Tabela 2). De acordo com a espécie de pitaya, a coloração da polpa dos frutos pode se diferenciar entre branca e vermelha. Portanto, analisar a coloração da polpa nos proporciona a identificação do grau de maturação da fruta, da quantidade de pigmentos presentes, se há interferência das diferentes formas de armazenamento e também pode ser um grande indicativo da aceitação pelo consumidor, visto que a cor polpa se torna um atrativo, devido a ampla diversidade de utilização, podendo tanto ser consumida in natura quanto no processamento de sucos, geleias, entre outros produtos.

Em trabalho realizado por Fernandes et al. (2017), os valores médios de ângulo hue na polpa branca de pitaya (Hylocereus undatus) foi de 94,01. Segundo Le Bellec et al. (2006), frutos de pitaya de polpa branca, como H. undatus apresentam polpa esbranquiçada com elevado número de sementes pequenas e negras.

Com relação aos sólidos solúveis, a polpa e a casca apresentaram valores médios de 8,31 e 4,50 ${ }^{\circ}$ Brix, respectivamente (Tabela 3). Os resultados obtidos são semelhantes aos observados por Abreu et al. (2012), onde a polpa e a casca apresentaram $10,83^{\circ}$ e $3,66^{\circ}$ brix, respectivamente.

Tabela 3. Características químicas de polpa e casca de pitaya 'Golden'. Pelotas-RS, 2017.

\begin{tabular}{lcccc}
\hline \multicolumn{1}{c}{ Variáveis analisadas } & Média & Mínimo & Máximo & CV (\%) \\
\hline SS ( ${ }^{\circ}$ brix) polpa & 8,31 & 5,70 & 10,90 & 21,76 \\
SS ( ${ }^{\circ}$ brix) casca & 4,50 & 3,60 & 5,90 & 16,09 \\
pH polpa & 4,79 & 4,28 & 5,22 & 6,41 \\
pH casca & 5,02 & 4,57 & 5,24 & 4,02 \\
AT (\% ácido málico) casca & 0,34 & 0,20 & 0,57 & 40,88 \\
AT (\% ácido málico) polpa & 0,18 & 0,13 & 0,25 & 0,04 \\
\hline
\end{tabular}

CV (\%): coeficiente de variação.

Fonte: Bruna Andressa dos Santos Oliveira. 
O teor de sólidos solúveis é uma medida indireta do teor de açúcares, pois aumenta à medida que esses teores se acumulam na fruta, o que ocorre com o amadurecimento devido à degradação dos polissacarídeos, e é um bom indicador do grau de maturação e sabor das frutas (Rangel Junior et al., 2021).

No que se refere ao $\mathrm{pH}$, verificou-se baixa variação entre as duas variáveis com valores médios de 4,79 para a polpa e 5,02 para a casca (Tabela 3). Lima et al. (2010), verificou média de 4,87 para o pH da polpa em pitaya (Hylocereus undatus). Entretanto, é importante ressaltar que podem ocorrer variações nos valores de $\mathrm{pH}$, em decorrência das características edafoclimáticas e genéticas.

Conforme Lima (2013), o pH é um parâmetro importante do fruto uma vez que pode influenciar o tempo de deterioração através do desenvolvimento de microrganismos, a atividade das enzimas, a retenção do sabor, o odor de produtos à base de frutas, a estabilidade de corantes artificiais em produtos de frutas, a verificação do estádio de maturação de frutas, a escolha da embalagem, a palatabilidade, a definição da temperatura de tratamento térmico, a escolha do tipo de material de limpeza e desinfecção, a escolha do equipamento com o qual se vai trabalhar na indústria, além da escolha de aditivos e conservantes.

Quanto à acidez titulável, a casca e a polpa apresentaram médias de $0,34 \%$ e $0,18 \%$ de ácido málico, respectivamente (Tabela 3). Resultados semelhantes foram registrados por Abreu et al. (2012), em que verificaram teor de acidez para a casca de $0,52 \mathrm{mg}$ de ácido cítrico. $100 \mathrm{~g}^{-1}$, e para a polpa $0,20 \mathrm{mg}$ de ácido cítrico. $100 \mathrm{~g}^{-1}$ em pitaya de polpa branca (Hylocereus undatus).

Segundo Frölech (2018), os teores de sólidos solúveis e acidez titulável são de suma importância no mercado de mesa, pois são indicadores de maturidade da fruta, influenciando diretamente em sua qualidade e, consequentemente, no sabor.

Em suma, através da caracterização físico-química da polpa e da casca de pitaya 'Golden' nas condições em que o experimento foi realizado verificou-se seu potencial para a elaboração de novos produtos, principalmente no que se refere ao aproveitamento da casca, contribuindo para redução do desperdício e dos danos ao meio ambiente, além de proporcionar uma maior geração de renda.

\section{Conclusão}

A polpa e a casca da pitaya 'Golden' apresentam características físico-químicas de acordo com a literatura, possuindo potencial tanto para o consumo in natura quanto para a utilização na indústria, bem como proporções de tamanho adequadas para o mercado externo.

\section{Agradecimentos}

À Coordenação de Aperfeiçoamento de Pessoal de Nível Superior (CAPES) e ao Conselho Nacional de Desenvolvimento Científico e Tecnológico (CNPq), pela concessão das bolsas de estudo e apoio financeiro à pesquisa.

\section{Referências}

Abdi, N. \& Mizrahi, Y. (2012). Effects of methyl bromide and storage time on postharvest behavior of three different cultivars of pitaya fruit, Israel Journal of Plant Sciences, 60(3), 319-324.

Abreu, W. C. De., Lopes, C. De O., Pinto, K. M., Oliveira, L. A., Carvalho, G. B. M. De., \& Barcelo, M. De F. P. (2012). Características físico-químicas e atividade antioxidante total de pitaias vermelha e branca. Revista Instituto Adolfo Lutz, 71 (4), 656-661.

Amid, M., \& Manap, M. Y. A. (2014). Purification and characterisation of a novel amylase enzyme from red pitaya (Hylocereus polyrhizus) peel. Food chemistry, 165, 412-418.

Campos-Rojas, E., Pinedo-Espinoza, J. M., Campos-Montiel, R. G., \& Hernandez-Fuentes, A. D. (2011). Avaliação de plantas pitaya (Stenocereus spp) de populações naturais de Monte Escobedo, Zacatecas. Revista Chapingo, 17(3), 173-182. 
Chitarra, M. I. F., \& Chitarra, A. B. (2005). Pós-colheita de frutas e hortaliças: fisiologia e manuseio. (2ª .ed.) Lavras: UFLA, 785p.

Cordeiro, M. H. M., Silva, J. M. D., Mizobutsi, G. P., Mizobutsi, E. H., \& Mota, W. F. D. (2015). Physical, chemical and nutritional characterization of pink pitaya of red pulp. Revista Brasileira de Fruticultura, 37(1), 20-26.

Donadio, L. C. (2009). Pitaya. Revista Brasileira de Fruticultura, 31(3), 637-929.

Fernandes, L. M. de S., Vieites, R. L., Lima, G. P. P., de Lima Braga, C., \& do Amaral, J. L. (2017). Caracterização do fruto de pitaia orgânica. Biodiversidade, 16(1), 167-178.

Frölech, D. B. (2018). Evolução da maturação, análise físico-química e sensorial de uvas e sucos de videiras Vitis labrusca e híbridas. Dissertação de mestrado, Universidade Federal de Pelotas, Pelotas, RS, Brasil.

Gunasena, H. P. M., Pushpakumara, D. K. N. G., \& Kariyawasam, M. (2007). Dragon Fruit Hylocereus undatus (Haw.) Britton and Rose. In: Pushpakumara, D. K. N., Gunasena, H. P.M., Singh, V. P. (Eds.) Underutilized fruit trees in Sri Lanka. World Agroforesty Centre, South Asia Office: India, 110-142.

Instituto Adolfo Lutz. (1985). Normas analíticas do Instituto Adolfo Lutz: métodos químicos e físicos para análise dos alimentos. (3a.ed.,) v.1. São Paulo: Instituto Adolfo Lutz, 371 p.

Junqueira, K. P., Junqueira, N. T. V., Ramos, J. D., \& Pereira, A. V. (2002). Informações preliminares sobre uma espécie de Pitaya do Cerrado. Documentos/ EMBRAPA Cerrados, Ed. 1. Planaltina, DF, 18p.

Le Bellec, F., Villant, F., \& Imbert, E. (2006). Pitahaia (Hylocereus spp.): a new crop, a market with a future. Fruits, France, 61(4), 237 - 250.

Lima, C. A. (2013). Caracterização, propagação e melhoramento genético de pitaya comercial e nativa do Cerrado. Tese de doutorado, Universidade de Brasília, Brasília, DF, Brasil.

Lima, C. D., Cohen, K. D. O., Faleiro, F. G., Junqueira, N. T. V., Bellon, G., Castelo Branco, M. T., Fuhrmann, E., Leão, A. J. P., \& De Oliveira, R. R. (2010). Caracterização físico-química e de compostos funcionais em frutos de pitaya. In Embrapa Cerrados-Artigo em anais de congresso (ALICE). In: Congresso Brasileiro De Fruticultura, Natal, RN, Brasil.

Magalhães, D. S. (2017). Desenvolvimento e maturação de frutos de pitaia vermelha de polpa branca. Tese de doutorado, Universidade Federal de Lavras, Lavras, MG, Brasil.

Marques, V. B., Moreira, R. A., Ramos, J. D., Araújo, N. A., \& Silva, F. O. R. (2011). Fenologia reprodutiva de pitaia-vermelha no município de LavrasMG. Ciência Rural, 41(6), 984-987.

Mello, F. R. (2014). Avaliação das características físico-químicas e atividade antioxidante da pitaya e determinação do potencial do mesocarpo como corante natural para alimentos. Tese de doutorado, Universidade Federal do Paraná, Curitiba, PR, Brasil.

Mello, F. R., Bernardo, C., Dias, C. O., Gonzaga, L., Amante, E. R., Fett, R., \& Candido, L. M. B. (2015). Antioxidant properties, quantification and stability of betalains from pitaya (Hylocereus undatus) peel. Ciência Rural, 45(2), 323-328.

Mercado-Silva, E. M. (2018). Pitaya-Hylocereus undatus (Haw)ExoticFruits:ReferenceGuide, 339-349p.

Minolta. (2007). Precise color communication: color control from perception to Instrumentation. Japan: Minolta Co. Ltd., 59 p.

Mizrahi, Y. (2014). Vine-cacti pitayas - the new crops of the world. Revista Brasileira de Fruticultura, 36(1), 124-138.

Moreira, R. A., Ramos, J. D., Araújo, N. A., \& Marques, V. (2011). Produção e qualidade de frutos de Pitaia-vermelha com adubação orgânica e granulado bioclástico. Revista Brasileira de Fruticultura, 33(1), 762-766.

Moreno, M. B., Barreto, C. F., Silva, P. S., Malgarim, M. B., \& Fachinello, J. C. (2016). Qualidade de pêssegos 'Maciel' sob armazenamento refrigerado com a utilização de embalagens. Revista Iberoamericana de Tecnología Postcosecha, 17(1), 58-64.

Oliveira, F. M., Oliveira, R. M., Maciejewski, P., Ramm, A., Manica-Berto, R., \& Zambiazi, R. C. (2017). Aspectos físico-químicos de geleia de pitaia em comparação com geleias de outras frutas vermelhas. Revista da Jornada de Pós-Graduação e Pesquisa-Congrega Urcamp, $1417-1423$.

Queiroz, M. I., \& Treptow, R. O. (2006). Análise Sensorial para a Avaliação da Qualidade dos Alimentos. Rio Grande: Ed. Da FURG, 268p.

Rangel Junior, I. M., Magalhães, D. S., Rodrigues, F. A., Pasqual, M., \& Pio, L. A. S. (2021). Qualidade de frutos e determinação do ponto de colheita de pitaia de polpa branca. Research, Society and Development, 10(7), p. e11810716287.

Rosales-Bustamante, E. P., Luna-Morales, C. D., \& Cruz-Leon, A. (2009). Classificação e seleção tradicional de pitaya (Stenocereus pruinosus (Otto)Buxb.) Em Tianguistengo, Oaxaca, e variação morfológica de cultivares. Revista Chapingo, 15, 75-82.

Sá, A. S. C. (2015). Caracterização química de bebidas fermentadas de pitaia (H. Undatus) cultivada no semiárido nordestino. Monografia de especialização, Instituto Federal de Educação, Ciência e Tecnologia do Sertão Pernambucano, Petrolina, PE, Brasil.

Santos, F. S., Araújo, K. T. A., Figueirêdo, R. M. F., Queiroz, A. J. D. M., \& Santiago, V. M. S. (2015). Cinética de secagem da casca da pitaya vermelha (Hylocereus undatus). Congresso Técnico Científico da Engenharia e da Agronomia, Fortaleza.

Sarmento, J. D. A. (2017). Qualidade, Compostos Bioativos E Conservação Da Pitaia (Hylocereus Polyrhizus) no Semiárido Brasileiro. Tese de doutorado, Universidade Federal Rural do Semi-Árido, Mossoró, RN, Brasil.

Schwentesius, R. R., \& Gómez, C. M. A. (1999). Perspectivas para a comercialização e exportação de frutas exóticas mexicanas nomercado internacional. CIESTAAM. Universidade Autônoma de Chapingo. México. 60p.

Silva, A. C. C. (2014). Pitaya: Melhoramento e produção de mudas. Tese de doutorado, Universidade Estadual Paulista, Jaboticabal, SP, Brasil. 
Research, Society and Development, v. 10, n. 14, e550101422540, 2021

(CC BY 4.0) | ISSN 2525-3409 | DOI: http://dx.doi.org/10.33448/rsd-v10i14.22540

Souza, L. S., Junqueira, K. P., Lima, C. A., Castiglioni, G. L., Villanova, A. C. C., Junqueira, N. T. V., Silva, D. G. P., Faleiro, F. G., Santos, E. C., Bellon, G., \& Junqueira, L. P. (2006). Características físico-químicas de frutos de pitaya cultivada nos cerrados. In: Congresso Brasileiro De Fruticultura, 19., 2006, Cabo Frio. Frutas do Brasil: saúde para o mundo: palestras e resumos. Cabo Frio: SBF; UENF; UFRRJ, 323p.

Surdo, S., \& Hickmann, F. (2019). Uso de farinha de casca da pitaya vermelha como fonte de fibras em macarrão fresco. In: XXXI Salão De Iniciação Científica da UFRGS, 2019, Campus do Vale. Resumos...Campus do Vale: Universidade Federal do Rio Grande do Sul, RS, Brasil.

Tenore, G. C., Novellino, E., \& Basile, A. (2012). Nutraceutical potential and antioxidant benefits of red pitaya (Hylocereus polyrhizus) extracts. Journal of functional foods, 4(1), 129-136.

Utpott, M., Krigger, S., Dias, C. Z., Thys, R. C. S., Rios, A. O., \& Flôres, S. H. (2019). Utilização da farinha da casca de pitaya vermelha (Hylocereus polyrhizus) como substituto de gordura em pães de forma. In: $6^{\circ}$ Simpósio de Segurança Alimentar, 2019, Gramado. Resumos...Gramado, Universidade Federal do Rio Grande do Sul, RS, Brasil.

Yah, A. R. C., Pereira, S. S., Veloz, C. S., Sañudo, R. B., \& Duch, E. S. (2008). Cambios físicos, químicos y sensoriales en frutos de pitahaya (Hylocereus undatus) durante su desarrollo. Revista Fitotecnia Mexicana, 31(1), 1-5. 